\title{
Acide linoléique conjugué et cancer du sein
}

\author{
Philippe BOUGNOUX ${ }^{1}$ \\ Aurélia BARASCU ${ }^{1}$ \\ Flore LAVILLONNIÈRE ${ }^{1}$ \\ Marie-Lise JOURDAN ${ }^{1}$ \\ Virginie MAILLARD ${ }^{1}$ \\ Véronique $\mathrm{CHAIES}^{1}$ \\ Jean-Louis SÉBÉDIO²
}

${ }^{1}$ INSERM E 0211, Université François Rabelais, CHU de Tours, Hôpital Bretonneau, 37044 Tours Cedex

${ }^{2}$ Laboratoire de nutrition lipidique, INRA, Dijon

\section{Introduction}

Les dérivés diène-conjugués de l'acide linoléique correspondent à une famille $d^{\prime}$ isomères géométriques et positionnels de l'acide linoléique (isomères diène-conjugués) collectivement dénommés CLA (pour conjugated linoleic acid). Ce groupe d'acides gras, présent dans les aliments d'origine animale comme la viande de ruminants ou dans certains produits laitiers [1], est également généré au cours de la cuisson [2], ainsi que sous l'action de la flore bactérienne intestinale [3]. L'isomère principal est le 9-cis,11-trans CLA. Cet isomère résulte aussi de la conversion endogène de l'acide vaccénique trans-11 $18: 1$, également présent dans le lait.

Les dérivés conjugués de l'acide linoléique (CLA) semblent avoir des propriétés inhibitrices de la cancérogenèse, notamment mammaire. L'utilisation de différentes espèces animales (souris, rates, hamsters), de diverses lignées cellulaires cancéreuses, et de plusieurs modèles de cancérogenèse (cancers chimio-induits avec des cancérigènes directs ou indirects, tumeurs transplantées) ont permis de confirmer le rôle protecteur des CLA en cancérogenèse pour différents sites: peau [2], estomac [4], pancréas [5], foie [6], poumon [7], glandes mammaires [8-11] et colique [11-15]. II a été rapporté un effet inhibiteur des CLA aux différentes phases : initiation $[9,16,17]$, promotion et croissance tumorales $[8,16]$, formation de métastases $[18,19]$.

Dans le modèle de tumeurs mammaires chimio-induites, des rates prépubères dont le régime était supplémenté en CLA avant l'initiation par un agent cancérigène (diméthylben$\mathrm{z}$ (a)anthracène ou N-nitroso-N-méthylurée), ont développé significativement moins de tumeurs que les rates témoins $(47 \%)[9,20]$, suggérant que la présence de CLA dans les tissus mammaires les rendraient moins sensibles à l'initiation ultérieure par un agent cancé- rigène. De même, si la supplémentation en CLA était réalisée après l'initiation par un agent cancérigène pendant 20 semaines, les rates développent significativement moins de tumeurs que les rates du lot témoin (32 à $60 \%$ de tumeurs en moins) $[8,9]$. Ces effets inhibi-

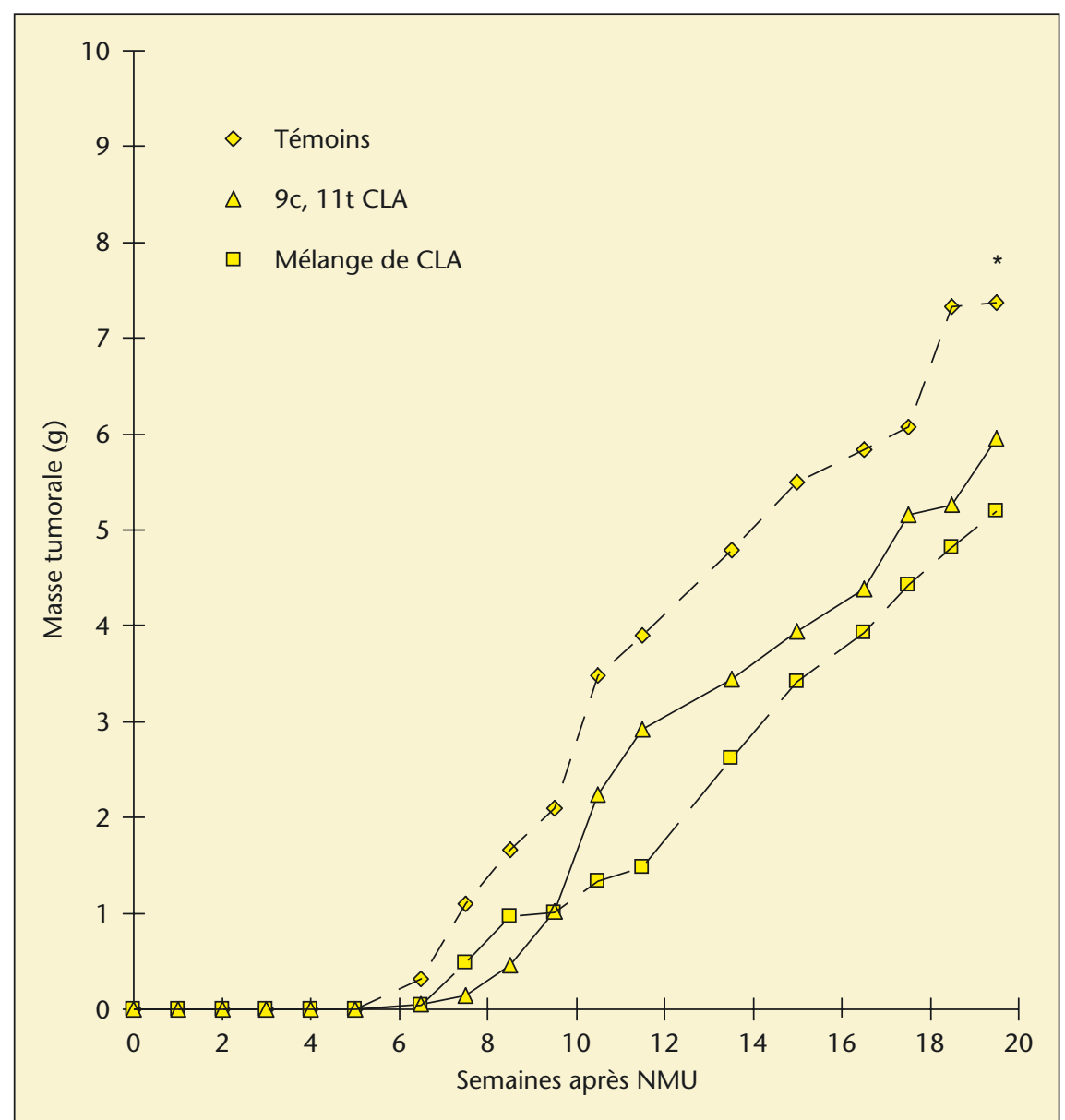

Figure 1. Masse tumorale mammaire chez le rat, estimée en fonction du régime. Trois groupes de rats SpragueDawley ont reçu au décours d'une initiation par NMU une diète comportant $5 \%$ d'huile de tournesol et une supplémentation de 1\% sous forme d'acides gras libres provenant soit de l'huile de tournesol (témoins), soit d'un mélange de CLA ou de l'isomère purifié $9 c, 11$. teurs de la promotion tumorale sont indépendants de la nature de l'agent cancérigène utilisé ou des lipides (nature et quantité) administrés dans le régime [21, 22]. L'apport de CLA dans la diète sous la forme d'acides gras libres semble avoir un effet similaire à celui des 
CLA sous forme de triglycérides [9], bien que I'accumulation de CLA dans la glande mammaire soit inférieure [23].

\section{Les CLA inhibent la cancérogenèse mammaire chez le rat}

Comme la majorité des données expérimentales provient d'une seule équipe [8,9,20-24], il a semblé utile de déterminer si des résultats similaires pouvaient être retrouvés de façon indépendante. De plus, comme chacun des isomères constitutifs des CLA est porteur d'une activité distincte de celle du mélange naturel, il était nécessaire d'examiner l'activité de l'isomère principal 9-cis,11-trans des CLA. Dans ce but, nous avons réalisé une série d'études $d$ 'intervention nutritionnelle chez le rat. Ces études ont été conçues pour comparer les effets de cet isomère à ceux d'un mélange $d$ 'isomères de CLA sur l'incidence, la multiplicité et la croissance de tumeurs mammaires autochtones induites chez le rat femelle par le $\mathrm{N}$-nitroso-N-méthylurée. Après l'étape d'initiation, les rats ont été séparés en trois groupes expérimentaux recevant un régime de base (5\% de lipides) supplémenté avec soit l'isomère 9-cis,11-trans synthétisé, soit un mélange $d$ 'isomères de CLA, soit des acides gras libres préparés à partir d'huile de tournesol pour le groupe témoin. On a observé, à 20 semaines d'évolution, une diminution de 30 et $35 \%$ du nombre de tumeurs/rat et une diminution de 44 et $45 \%$ de la masse tumorale/rat dans les groupes ayant reçu respectivement le mélange de CLA et l'isomère 9-cis,11-trans de synthèse (figure 1). L'incorporation tissulaire des CLA a été mesurée lors du sacrifice. La mesure des taux d'isomères de CLA a montré que ces isomères avaient bien été incorporés dans le tissu adipeux mammaire et dans les tissus tumoraux (tableau 1). Ainsi, le principal isomère de CLA présent dans I'alimentation est inhibiteur en carcinogenèse mammaire, comme l'est le mélange de CLA [25].

\section{CLA et risque de cancer du sein}

II n'existe que peu de données concernant la relation entre les apports alimentaires de CLA et le risque de cancer du sein, principalement en raison de l'absence de disponibilité de questionnaires permettant une estimation des apports alimentaires. Pour déterminer si les CLA protègent contre le risque de cancer du sein, nous avons réalisé une étude cas-témoins à partir d'une population de 213 patientes présentant une tumeur du sein maligne, invasive mais non métastatique (cas), et de 84
Tableau 1. Incorporation tissulaire en CLA après 16 semaines d'intervention nutritionnelle chez le rat.

\begin{tabular}{|lccc|}
\hline Site & \multicolumn{3}{c|}{ Groupes nutritionnels } \\
\cline { 2 - 4 } & Témoin & Mélange de CLA & 9c,11t-CLA \\
\hline Tissu adipeux mammaire & $0,18^{\mathrm{a}}$ & $5,64^{\mathrm{b}}$ & $6,53^{\mathrm{c}}$ \\
Phospholipides de tumeur* & 0,03 & 0,40 & 0,26 \\
\hline
\end{tabular}

$\%$ des acides gras totaux, moyenne de 5 animaux/groupes; $a, b, c=p<0,05$. patientes avec une tumeur du sein bénigne (témoins). Nous avons utilisé le taux de CLA du tissu adipeux comme biomarqueur des apports alimentaires passés de CLA. Le taux de CLA a été mesuré dans un fragment biopsique de tissu adipeux conservé lors de la chirurgie. Le taux moyen de CLA représentait 0,44\% de I'ensemble des acides gras du tissu adipeux, téristiques des deux populations étudiées sont présentées dans le tableau 2. La population de cas était plus âgée, une proportion plus élevée de femmes ménopausées et l'indice de masse corporelle était plus élevé que dans la population témoin. Un modèle de régression logistique non conditionnelle a été utilisé pour obtenir l'estimation des Odds ratio, après ajustement sur l'âge, le statut ménopausique et I'indice de masse corporelle [26]. Nous n'avons pas trouvé d'association significative entre les CLA du tissu adipeux et le risque de cancer du sein (tableau 3). allant de $0,14 \%$ à $0,75 \%$ (figure 2). Les carac-

\section{CLA et récidives des cancers du sein}

Comme les CLA inhibent la croissance tumorale chez le rat, il a semblé intéressant d'examiner ce point chez l'Homme. Le cancer du sein est une situation appropriée, puisque la tumeur réapparaît au décours du traitement, dans un site distant (métastase), dans environ un tiers des cas. Pour cette raison, la relation entre le taux de CLA présent dans le tissu adipeux au moment du diagnostic initial, et la survenue ultérieure de métastases a été examinée dans la population des patientes porteuses d'un cancer du sein. Aucun lien n'a été observé entre le taux de CLA et les facteurs pronostiques du cancer du sein, i.e. : taille de la tumeur, atteinte des ganglions axillaires, grade histopronostique, réceptivité hormonale (figure 3 ) ni avec l'index mitotique ou la présence d'emboles vasculaires. II n'est pas non plus apparu de relation significative avec le risque de récidive ou de

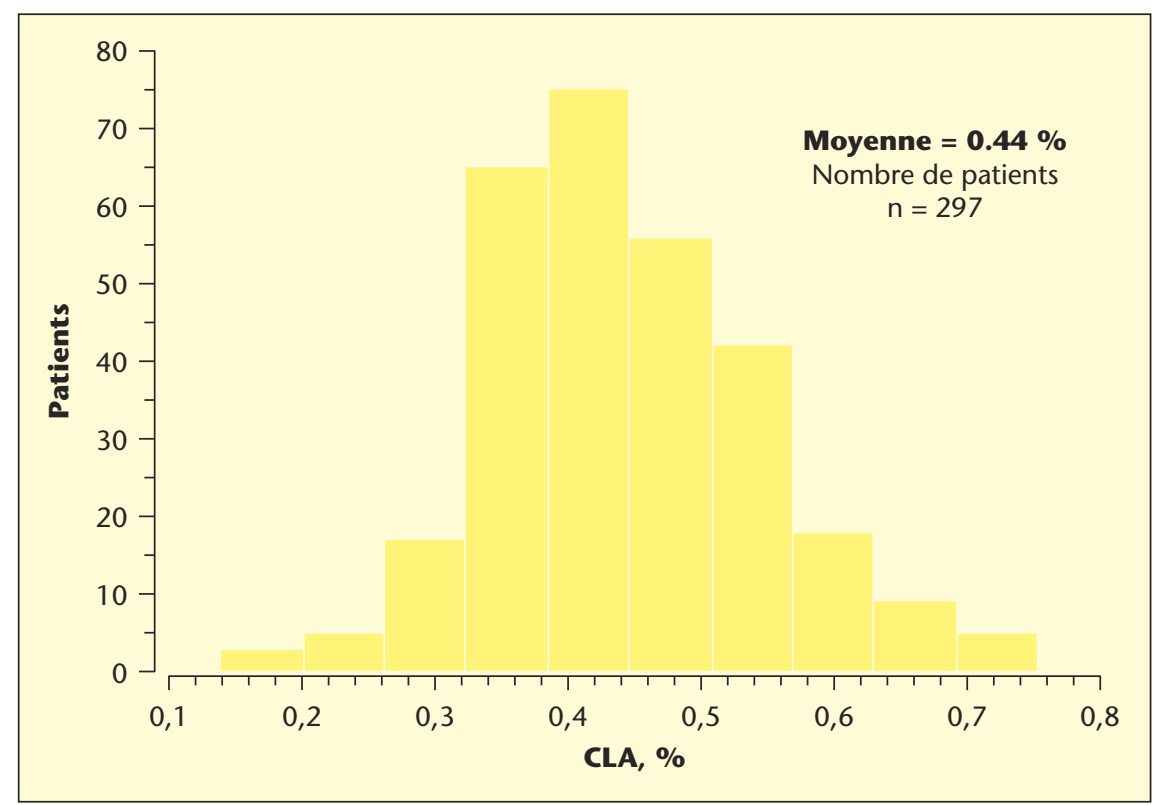

Figure 2. Distribution des patientes en fonction du taux de CLA dans le tissu adipeux mammaire. Un fragment de tissu adipeux prélevé lors de l'acte chirurgical diagnostique chez 297 patientes opérées pour une tumeur mammaire bénigne ou maligne a été analysé. Les lipides ont été extraits, purifiés, et les CLA quantifiés (Lavillonnière et al., 2003). 
Tableau 2. Caractéristiques de la population.

\begin{tabular}{|lccc|}
\hline & Témoin $(\mathrm{n}=84)$ & Cas $(\mathrm{n}=213)$ & $\mathrm{p}^{\mathrm{a}}$ \\
\hline $\begin{array}{l}\text { Âge (années) } \\
\text { Moyenne (SE) }\end{array}$ & $42.7(1.5)$ & $55.4(0.9)$ & 0.0001 \\
$\mathrm{IMC}\left(\mathrm{kg} / \mathrm{cm}^{2}\right)$ & $22.4(0.4)$ & $24.5(0.3)$ & 0.0009 \\
Moyenne (SE) & & & \\
Statut ménopausique (\%) & 77.4 & 41.8 & 0.001 \\
Pré-ménopause & 22.6 & 58.2 & \\
Post-ménopause & & & \\
\hline
\end{tabular}

IMC : Indice de masse corporelle $;{ }^{a}$ test chi ${ }^{2}$ ou Student-t.

Tableau 3. Risque relatif estimé de cancer du sein (Ors, brut et ajusté*), et intervalle de confiance $95 \%$ en fonction du taux de CLA dans le tissu adipeux de la population dans son ensemble $(n=297)$.

\begin{tabular}{|lcccc|}
\hline Acide gras & \multicolumn{3}{c|}{ ORs $^{\mathrm{a}}(95 \% \mathrm{ICs})$} & \multirow{2}{*}{ P de tendance } \\
\cline { 2 - 4 } & Tertile 1 (bas) & Tertile 2 & Tertile 3 (élevé) & \\
\hline CLA & 1,00 & $1,29(0,68-2,45)$ & $1,42(0,77-2,65)$ & 0,27 \\
& & $1,65(0,80-3,37)^{*}$ & $1,83(0,90-3,71)^{*}$ & 0,10 \\
\hline
\end{tabular}

*Ajusté sur l'âge au diagnostic, l'indice de masse corporelle et le statut ménopausique.

métastases (figure 4). Ainsi, dans une cohorte de patientes traitées pour un cancer du sein, il n'a pas été observé de relation entre le CLA dans les tissus de réserve et la recroissance tumorale au cours du suivi [27].

\section{Discussion et perspectives}

Si les données disponibles n'indiquent pas que les CLA alimentaires influencent le risque de cancer du sein ou son évolution métastatique, elles n'éliminent pas la possibilité que les CLA puissent être protecteurs en cancérogenèse mammaire humaine. La raison pourrait être le faible taux de CLA disponibles dans l'alimentation humaine, au moins dans la région Centre de la France, où cette étude a été réalisée. En effet, le taux moyen dans le tissu adipeux chez l'homme est à peine plus élevé que celui observé chez le rat non supplémenté, et beaucoup plus bas (plus de 10 fois) que le taux présent chez le rat recevant une supplémentation de $1 \%$ en CLA. Or si les effets inhibiteurs de la promotion tumorale s'observent dès 0,1\% de CLA dans le régime alimentaire, ils dépendent linéairement de la dose apportée jusqu'à $1 \%$ (en poids) de la diète $[8,10]$.

Ainsi nos deux études, cas-témoins et pronostique, qui ont comparé entre elles des populations de patientes ayant des apports de CLA trop faibles pour qu'un effet bénéfique puisse être apparent, ne permettent pas de conclure. Ces molécules, présentes dans de nombreux produits alimentaires pourraient être actives, mais à des concentrations bien supérieures à celles apportées dans notre alimentation, contrairement à ce qui avait été précédemment évoqué $[20,24]$. On ne sait pas actuellement si une supplémentation en CLA dans des conditions permettant d'augmenter les taux de
CLA dans les tissus aurait un effet bénéfique chez I'homme, ni si une telle supplémentation serait réalisable dans des conditions de sécurité nécessaires. Pour autant, les enjeux restent élevés, puisque ces molécules restent candidates à une prévention nutritionnelle du cancer du sein. Une modélisation de cancérogenèse mammaire dans une espèce animale de taille intermédiaire entre le rat et l'Homme pourrait permettre de déterminer s'il serait légitime de prendre un tel risque chez l'Homme. Dans cette perspective, I'utilisation d'animaux domestiques, espèces connues pour développer spontanément des tumeurs mammaires durant la deuxième partie de leur vie, pourrait être considérée pour une telle intervention nutritionnelle ciblée sur les CLA à doses élevées.

Remerciements. Les travaux cliniques ont été possibles grâce à O. Le Floch, J. Lansac, G. Body, et le personnel du bloc opératoire du service de gynécologie du CHU de Tours, et à C. Couet qui a apporté les compétences de nutrition humaine. F. Fetissof, T. Lefranca et MC Grangeponte ont réalisé les études anatomopathologiques, et M. Pinault, pour son aide logistique. Les supports financiers ont été apportés par la Région Centre et le CERIN (FL, bourse cofinancée), le CHU de Tours (VC) et les comités départementaux de la Ligue Nationale Contre le Cancer (Comités du Cher, de la Charente, du Loir-et-Cher et de I'Indre-etLoire). Nous souhaitons associer à l'ensemble de ces travaux notre collègue Claude Lhuillery, INRA, Jouy-en-Josas.

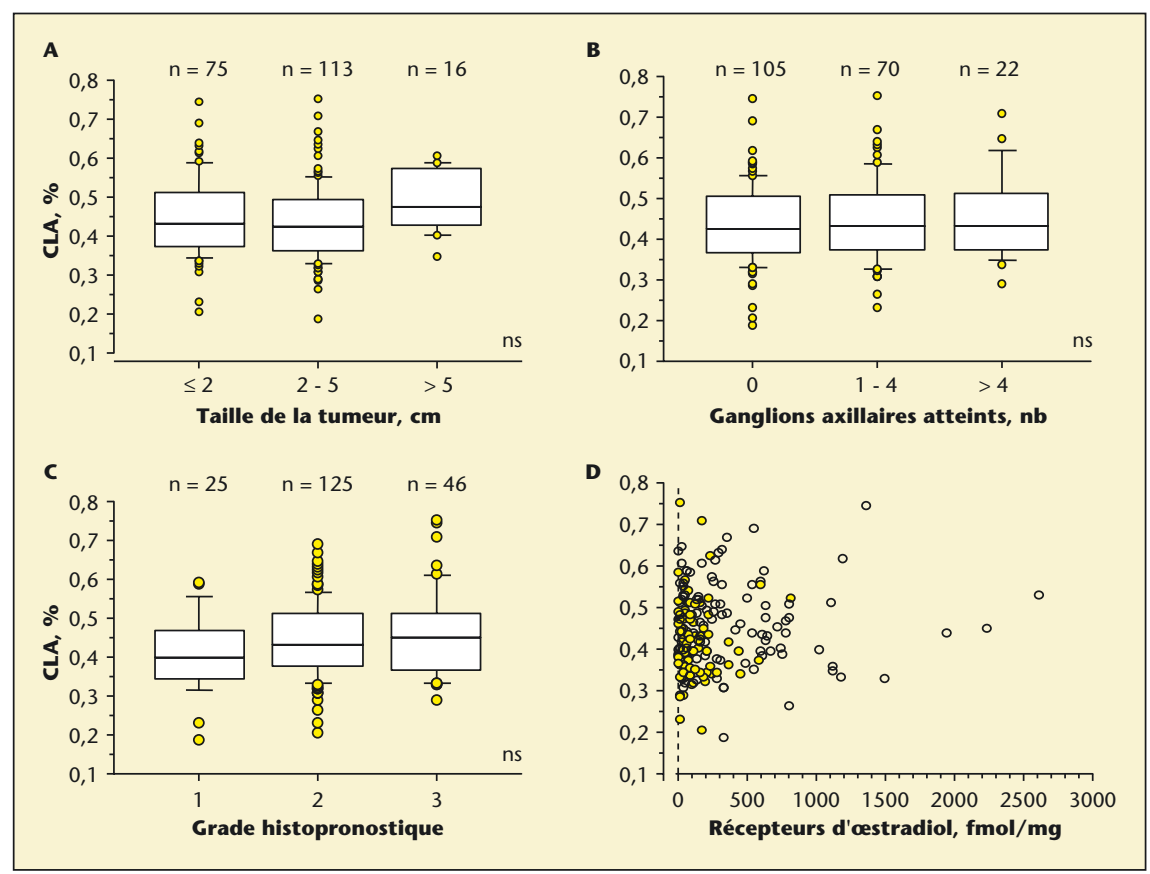

Figure 3. Facteurs pronostiques du cancer du sein et réserves en CLA du tissu adipeux mammaire. A) Taux de CLA du tissu adipeux mammaire selon la taille de la tumeur lors du diagnostic de cancer $d u$ sein ; B) selon le degré d'envahissement ganglionnaire axillaire; C) selon le grade histopronostique de la tumeur ; D) selon la richesse en récepteurs d'oestradiol du tissu tumoral. Les cercles sont pleins ou vides selon le statut hormonal (non ménopausé ou ménopausé) des patientes lors du diagnostic. 


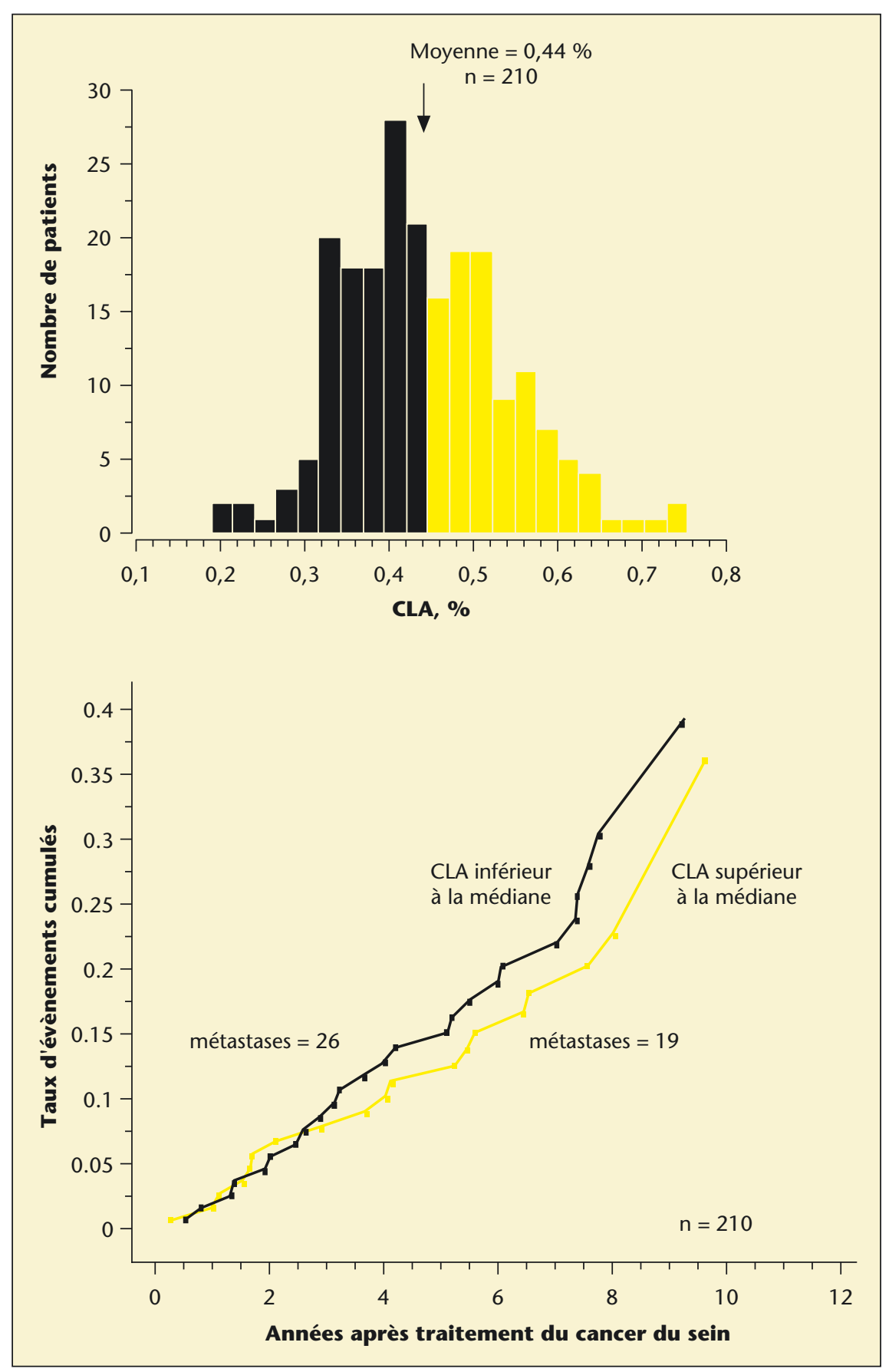

Figure 4. Récidives et métastases au cours du suivi chez des patientes traitées pour un cancer du sein, en fonction des réserves tissulaires en CLA. La population a été séparée en deux groupes selon que le taux de CLA du tissu adipeux mammaire prélevé au moment du diagnostic de cancer du sein était supérieur (gris clair) ou inférieur (noir) à la valeur médiane et le taux d'évènements cumulés a été calculé au cours du temps après traitement (figure du bas). Distribution des patientes selon le taux de CLA du tissu adipeux mammaire (figure du haut).

\section{RÉFÉRENCES}

1. HUANG Y-C, LUEDECKE LO, SCHULTZ TD. Effect of cheddar cheese consumption on plasma conjugated linoleic acid concentration in men. Nutr Res $1994 ; 14: 373-86$.
3. CHIN SF, STORKSON JM, LIU W, ALBRICHT KJ, PARIZA MW. Conjugated linoleic acid $(9,11$ and 10,12-octadecadienoic acid) is produced in conventional but not germ-free rats fed linoleic acid. / Nutr $1994 ; 124$ : 694-701.

4. HA YL, STORKSON J, PARIZA MW. Inhibition of benzo(a)pyrene-induced mouse forestomach neoplasia by conjugated dienoic derivatives of linoleic acid. Cancer Res $1990 ; 50$ : 1097-101.

5. APPEL MJ, VAN, GARDEREN-HOETMER AM, WOUTERSEN RA. Effects of dietary conjugated linoleic acid on pancreatic carcinogenesis in rats and hamsters. Cancer Res $1994 ; 54$ : 211320.

6. DESBORDES C, LEA MA. Effects of C18 fatty acid isomers on DNA synthesis in hepatoma and breast cancer cells. Anticancer Res 1995 ; $15: 2017-22$

7. SCHONBERG S, KROKAN HE. The inhibitory effect of conjugated dienoic derivatives (CLA) of linoleic acid on the growth of human tumor cell lines is in part due to increased lipid peroxidation. Anticancer Res $1995 ; 15$ : 1241-6.

8. IPC, CHIN SF, SCIMECA JA, PARIZA MW. Mammary cancer prevention by conjugated dienoic derivative of linoleic acid. Cancer Res $1991 ; 51: 6118-24$.

9. IP C, SINGH M, THOMPSON HJ, SCIMECA JA. Conjugated linoleic acid suppresses mammary carcinogenesis and proliferative activity of the mammary gland in the rat. Cancer Res 1994 ; $54: 1212-5$.

10. SHULTZ TD, CHEW BP, SEAMAN WR. Differential stimulatory and inhibitory responses of human MCF-7 breast cancer cells to linoleic acid and conjugated linoleic acid in culture. Anticancer Res $1992 ; 12$ : 2143-6.

11. CHENG IL, FUTAKUCHI M, OGAWA K, ET AL. Dose response study of conjugated fatty acid derived from safflower oil on mammary and colon carcinogenesis pretreated with 7,12dimethylbenz[a]anthracene (DMBA) and 1,2dimethylhydrazine $(\mathrm{DMH})$ in female SpragueDawley rats. Cancer Lett $2003 ;$ 196(2) : 161-8.

12. LIEW C, SCHUT $H, C H I N$ S, PARIZA M, DASHWOOD R. Protection of conjugated linoleic acids against 2-amino-3methylimidazo[4,5-f]quinoline-induced colon carcinogenesis in the $\mathrm{F} 344$ rat : a study of inhibitory mechanisms. Carcinogenesis $1995 ; 16$ : 3037-43.

13. COLEMAN LI, LANDSTROM EK, ROYLE PJ, BIRD AR, MCINTOSH GH. Protection of conjugated linoleic acids against 2-amino-3methylimidazo[4,5-f]quinoline-induced colon carcinogenesis in the $\mathrm{F} 344$ rat : a study of inhibitory mechanisms. Carcinogenesis $2002 ; 16$ : 3037-43.

14. KOHNO H, SUZUKI R, NOGUCHI R, HOSOKAWA M, MIYASHITA K, TANAKA T. Dietary conjugated linolenic acid inhibits azoxymethaneinduced colonic aberrant crypt foci in rats. Jpn J Cancer Res $2002 ; 93: 133-42$. 
15. KIM K, PARK HS. Dietary supplementation of conjugated linoleic acid reduces colon tumor incidence in $\mathrm{DMH}$-treated rats by increasing apoptosis with modulation of biomarkers. Nutrition 2003 ; 19(9) : 772-7.

16. BELURY MA, NICKEL KP, BIRD CE, WU Y. Dietary conjugated linoleic acid modulation of phorbol ester skin tumor promotion. Nutr Cancer 1996 ; 26 : 149-57.

17. ZU HX, SCHUT HAI. Inhibition of 2-amino-3methylimidazole(4,5-f)quinoline-DNA adduct formation in CDFI mice by heat altered derivatives of conjugated linoleic acid. Food Chem Toxicol $1992 ; 30: 9-16$

18. VISONNEAU S, CESANO A, TEPPER SA, SCIMECA IA, SANTOLI D, KRITCHEVSKY D. Conjugated linoleic acid suppresses the growth of human breast adenocarcinoma cells in SCID mice. Anticancer Res $1997 ; 17(2 A)$ : 969-73.
19. HUBBARD NE, LIM D, SUMMERS L, ERICKSON $\mathrm{KL}$. Reduction of murine mammary tumor metastasis by conjugated linoleic acid. Cancer Lett $2000 ; 150(1)$ : 93-100.

20. IP C, SCIMECA JA, THOMPSON HJ. Effect of timing and duration of dietary conjugated linoleic acid on mammary cancer prevention. Nutr Cancer $1995 ; 24: 241-7$.

21. IP C, BRIGGS SP, HAEGELE AD, THOMPSON H STORKSON J, SCIMECA JA. The efficacy of conjugated linoleic acid in mammary cancer prevention is independent of the level or the type of fat in the diet. Carcinogenesis $1996 ; 17(5)$ : 1045 50

22. IP C, SCIMECA JA. Conjugated linoleic acid and linoleic acid are distinctive modulators of mammary carcinogenesis. Nutr Cancer 1997 ; 27(2) : 131-5

23. IP C, BANNI S, ANGIONI E, ET AL. Conjugated linoleic acid-enriched butter fat alters mammary gland morphogenesis and reduces cancer risk in rats. / Nutr $1999 ; 29(12)$ : 2135-42.
24. IP C, SCIMECA JA, THOMPSON HJ. Conjugated linoleic acid, a powerful anticarcinogen from animal fat sources. Cancer 1994; 74 : 1050-4.

25. LAVILLONNIERE F, CHAIES V, MARTIN IC, SEBEDIO JL, LHUILLERY C, BOUGNOUX P. Dietary purified cis-9,trans-11 conjugated linoleic acid isomer has anticarcinogenic properties in chemically induced mammary tumors in rats. Nutr Cancer 2003 ; 45(2) : 190-4.

26. CHAIÈS V, LAVILLONNIÈRE F, FERRARI $P$, et al. Conjugated linoleic acid content in breast adipose tissue is not associated to the relative risk of breast cancer in a population of French patients. Cancer Epidemiol Biomarkers Prev $2002 ; 11: 672-3$.

27. CHAJÈS V, LAVILLONNIÈRE $F$, MAILLARD V, et al. Conjugated linoleic acid content in breast adipose tissue of breast cancer patients and the risk of metastasis. Nutr Cancer 2003; 45(1): 17-23. 